\title{
Adjacency stable connected operators and set levelings
}

\author{
Jose Crespo \\ GIB - LIA, Facultad de Informática, Universidad Politécnica de Madrid, Campus de \\ Montegancedo, Boadilla del Monte (Madrid), Spain \\ jcrespo@fi.upm.es
}

\begin{abstract}
This paper studies morphological connected operators. Particularly, it focuses on an adjacency constraint, as well as on the socalled set levelings. Two important findings are reported in this work. First, the relationships between the so-called adjacency stable operators and set levelings are investigated, and an equivalence is established. This is an important result about how these concepts have been chronologically introduced, and it permits to apply some properties to the related operator class. Second, the implications and limits of a property about expressing certain connected operators as a sequential composition of an opening and a closing (and vice-versa) based on markers are discussed. Then, a commutative property for attribute alternated filters is presented.
\end{abstract}

Keywords: image processing, image analysis, mathematical morphology, connected operator, adjacency stable operator, leveling.

\section{Introduction}

This paper investigates some aspects of morphological connected operators, which preserve well shapes and do not introduce discontinuities.

It happens that usual morphological connected operators (such as those composed by openings and closings) impose certain adjacency constraints between the input and the output. This was previously researched, and the so-called adjacency stable connected operator concept $[6,10,11]$ was established in the set or binary framework.

In addition, the so-called levelings [20-23] were defined in the non-binary framework in such a way that constrain the output variations depending on the input variations. Those levelings within the set or binary framework are called set or binary levelings [22].

A question that naturally arises is whether there are relationships between adjacency stable connected operators and set levelings. As will be shown later, this is one of the results that will be established in this work. In scientific research, it is key being able to indicate how and when research concepts have been developed and introduced. Besides, in the case where such relationships exist, properties that are valid for one type of operators could perhaps apply to the other related operator class. 
Proceedings of the $8^{\text {th }}$ International Symposium on Mathematical Morphology, Rio de Janeiro, Brazil, Oct. 10-13, 2007, MCT/INPE, v. 1, p. 215-226.

http://urlib.net/dpi.inpe.br/ismm@80/2007/03.01.11.36

A second aspect researched in this paper is the possibility of expressing certain connected operators as the sequential composition of an opening and a closing (and vice-versa). Some clarifications about the implications, and limits, of a previously presented result are provided. Furthermore, a commutative property for attribute alternated filters will be presented.

Section 2 provides some general background. The adjacency stability and leveling concepts are recalled in, respectively, Sections 3 and 4 . The relationships between adjacency stable connected operators and set levelings are established in Section 5. The expression of certain connected operators as sequential compositions of an opening and a closing is treated in Section 6 . Finally, a conclusion section ends the paper.

\section{Background}

\subsection{General definitions}

Mathematical morphology deals with the application of set theory concepts to image processing and analysis, and it considers that images are composed of geometrical shapes with intensity or multi-band profiles [25]. Some general references are $[1,13,15,16,19,29-31,35]$.

A basic set of notions on morphological filtering can be the following.

- Mathematical morphology focuses on increasing mappings defined on a complete lattice [31]. In a complete lattice there exists an ordering relation, and two basic operations called infimum and supremum (denoted by $\bigwedge$ and $\bigvee$, respectively).

- A transformation $\psi$ is increasing if and only if it preserves ordering.

- A transformation $\psi$ is idempotent if and only if $\psi \psi=\psi$.

- A transformation $\psi$ is a morphological filter if and only if it is increasing and idempotent.

- An opening (denoted by $\gamma$ ) is an anti-extensive morphological filter. Since $\gamma$ is anti-extensive, we can say that $\gamma \leq \mathrm{id}$, where id symbolizes the identity operator that leaves the input unchanged.

- A closing (denoted by $\varphi$ ) is an extensive morphological filter. Since $\varphi$ is extensive, we can say that $\varphi \geq \mathrm{id}$.

In the theoretical expressions in this paper, we will be working on the lattice $\mathcal{P}(E)$, where $E$ is a given set of points (the space) and $\mathcal{P}(E)$ denotes the set of all subsets of $E$ (i.e., $\mathcal{P}(E)=\{A: A \subseteq E\}$ ). In other words, inputs and outputs are supposed to be sets or, equivalently, binary functions. In this lattice, the sup $\bigvee$ and the $\inf \wedge$ operations are, respectively, the set union $\bigcup$ and set intersection $\bigcap$ operations, while the order relation is the set inclusion relation $\subseteq$. Even though we will work on the lattice $\mathcal{P}(E)$, results are extendable to gray-level functions by means of the so called flat operators $[30,35]$.

Two morphological operators $\psi_{1}$ and $\psi_{2}$ are dual if $\psi_{1}=\complement \psi_{2} \complement$, where $\complement$ symbolizes the complement operator. 
Proceedings of the $8^{\text {th }}$ International Symposium on Mathematical Morphology, Rio de Janeiro, Brazil, Oct. 10-13, 2007, MCT/INPE, v. 1, p. 215-226.

\subsection{Some background on connectivity and connected operators}

Some references to the topic of connectivity and connected filtering are: [2-5, 7, 9-12, 14, 17, 18, 20-24, 26-28, 32-38].

Connectivity is established in [31, (pp. 51-57)] by means of the connected class concept. A connected class $\mathcal{C}$ in $\mathcal{P}(E)$ is a subset of $\mathcal{P}(E)$ such that (a) $\emptyset \in \mathcal{C}$ and for all $x \in E,\{x\} \in \mathcal{C}$; and (b) for each family $C_{i}$ in $\mathcal{C}, \bigwedge_{i} C_{i} \neq \emptyset$ implies $\bigvee_{i} C_{i} \in \mathcal{C}$. The subclass $\mathcal{C}_{x}$ that has all members of $\mathcal{C}$ that contain $x$ (i.e., $\mathcal{C}_{x}=\{C \in \mathcal{C}: x \in C\}$ ) leads to the definition of an opening $\gamma_{x}$ called point opening [31]. For all $x \in E, A \in P(E)$,

$$
\gamma_{x}(A)=\bigvee\left\{C: C \in \mathcal{C}_{x}, C \leq A\right\} .
$$

The dual operation of $\gamma_{x}$ is the closing $\varphi_{x}$ that is equal to $\complement \gamma_{x} \complement$. If a point $x$ does not belong to a set $A$, i.e., it belongs to a pore of $A$, then we can obtain such a pore with $\gamma_{x} \complement$ (or, equivalently, with $\complement \varphi_{x}$ ).

In sets (or, equivalently, binary images), the flat zone of a point (or pixel) $x$ is the grain or the pore (whichever is not empty) which $x$ belongs to. I.e., the flat zone of $x$ in a set $A$ is equal to: $\mathrm{F}_{x}(A)=\gamma_{x}(A) \bigvee \gamma_{x} \complement(A)$. (Note that either the grain or the pore of a point $x$ is empty.) In the nonbinary case, the flat zones of a function are its piecewise-constant regions, i.e., the set of connected sets with the same function value.

An operator $\psi$ is connected $[28,34]$ if, for all $A \in P(E)$, each flat zone (grain or pore) of $A$ is included in a flat zone (grain or pore) of $\psi(A)$.

\subsection{Connectivity requirement}

A general requirement for the space connectivity is assumed in this work. Particularly, the space connectivity is supposed to be a strong connectivity (see $[17,24]$ ). The usual four- and eight-connectivities in connected subsets of $\mathbb{Z}^{2}$ are cases of strong connectivities.

\section{Adjacency stability}

This section discusses and summarizes the adjacency stability concept for connected operators, which appeared first in [11], and was further studied in $[6,10]$. A closely related concept was later discussed in [17].

Let us define the concept of adjacency between flat zones, which formalizes the intuitive notion of contiguity.

Definition 1. Two (disjoint) flat zones $\mathrm{F}_{x}(A)$ and $\mathrm{F}_{y}(A)$ in a space $E$ (endowed with $\gamma_{x}, x \in E$ ) are said to be adjacent if $\mathrm{F}_{x}(A) \bigvee \mathrm{F}_{y}(A)$ is a connected set, i.e., if $\mathrm{F}_{x}(A) \bigvee \mathrm{F}_{y}(A)=\gamma_{x}\left(\mathrm{~F}_{x}(A) \bigvee \mathrm{F}_{y}(A)\right)$.

(Note that $\mathrm{F}_{x}(A)=\gamma_{x}(A) \bigvee \gamma_{x} \complement(A)$.) The relationship of Definition 1 is symmetric (and not reflexive). Definition 1 can be extended to sets: two sets $A$ and $B$ are adjacent if some flat zone of $A$ is adjacent to some flat zone of $B$. 
Proceedings of the $8^{\text {th }}$ International Symposium on Mathematical Morphology,

Rio de Janeiro, Brazil, Oct. 10-13, 2007, MCT/INPE, v. 1, p. 215-226.

http://urlib.net/dpi.inpe.br/ismm@80/2007/03.01.11.36

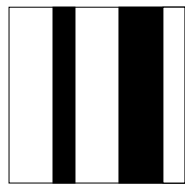

(a) Input set (in dark)

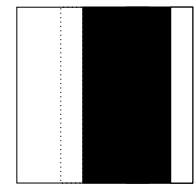

(b) AU Output

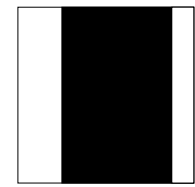

(c) AS Output

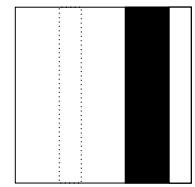

(d) AS Output

Figure 1. Adjacency stability example. Part (a) shows an input set $A$, and parts (b), (c) and (d) display three possible outputs of connected operators. Part (b) would be adjacency unstable: note that a pore of the input set $A$ is in a grain in (b) but is not surrounded by grains of $A$. This situation does not happen in cases (c) and (d). (Note: "AU" refers to adjacency unstable, and "AS" denotes adjacency stable.) (Note: the space is endowed with a usual four- or eight-connectivity.)

The adjacent flat zones of a point $x$ in an input set $A$, symbolized by $\mathrm{D}_{x}(A)$, are the pores (if $x \in A$ ) or the grains (if $x \notin A$ ) that are adjacent to $\mathrm{F}_{x}(A)$, i.e.,

$$
\mathrm{D}_{x}(A)=\bigvee_{y}\left\{\mathrm{~F}_{y}(A): y \in E, \mathrm{~F}_{y}(A) \bigvee \mathrm{F}_{x}(A)=\gamma_{x}\left(\mathrm{~F}_{y}(A) \bigvee \mathrm{F}_{x}(A)\right\}\right.
$$

The important concept of adjacency stability $[6,10,11]$ is established next. This requirement concerns how adjacent grains and pores are treated by an operation.

Definition 2. Let $E$ be a space endowed with $\gamma_{x}, x \in E$. An operator $\psi: \mathcal{P}(E) \Rightarrow \mathcal{P}(E)$ is adjacency stable if, for all $x \in E$ :

$$
\gamma_{x}(\mathrm{id} \bigvee \psi)=\gamma_{x} \bigvee \gamma_{x} \psi
$$

Note that $\gamma_{x}$ commutes under the $\inf \left(\gamma_{x}\left(\bigwedge_{i} \psi_{i}\right)=\bigwedge_{i} \gamma_{x} \psi_{i}\right)$ but not in general under the sup.

The adjacency stability equation 2 treats grains and pores symmetrically. The reason is that what matters is the switch from grain to pore and viceversa. We can state as well that the dual of an adjacency stable operator is adjacency stable.

The grain-pore relationship is illustrated in Figure 1. The consequences of adjacency stability on the relationships between the grains of an input set $A$ and the output $\psi(A)$ are the following: the grains of $\psi(A)$ are a union of (a) grains of $A$, and (b) pores of $A$ surrounded by grains in (a). For the particular case in Figure 1(b), Figure 2 shows that the adjacency stability equation does not hold for the point marked as $x$ (this point is not the only one). The adjacency stability equation must hold for all $A \in \mathcal{P}(E)$ and for all $x \in E$.

Lemma 1 is useful to relate the input and the output.

Lemma 1. Let $E$ be a space endowed with $\gamma_{x}, x \in E$. A connected operator $\psi: \mathcal{P}(E) \Rightarrow \mathcal{P}(E)$ is adjacency stable if and only if, for all $A \in \mathcal{P}(E), \psi(A)$ and $A \backslash \psi(A)$ are not connected to each other (i.e., are not adjacent). 
Proceedings of the $8^{\text {th }}$ International Symposium on Mathematical Morphology,

Rio de Janeiro, Brazil, Oct. 10-13, 2007, MCT/INPE, v. 1, p. 215-226.

http://urlib.net/dpi.inpe.br/ismm@80/2007/03.01.11.36

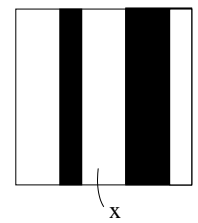

(a) Input set $A$ (in dark)

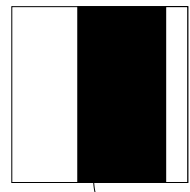

$\mathrm{x}$

(c) $\gamma_{x}(A) \bigvee \gamma_{x} \psi(A)$

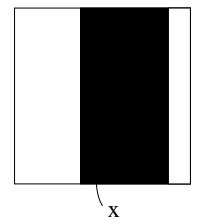

(b) $\psi(A)$ ( $\psi$ is adjacency unstable)

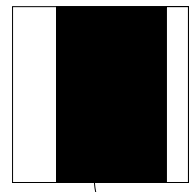

$\mathrm{x}$

(d) $\gamma_{x}\left(\mathrm{id} \bigvee \gamma_{x} \psi\right)(A)$

Figure 2. Adjacency stability equation. Parts (a) and (b) display, respectively, an input set $A$ and the output $\psi(A)$, where $\psi$ is an adjacency unstable connected operator. The adjacency stability equation does not hold: for the point $x$ signaled (among others), $\gamma_{x}(A) \bigvee \gamma_{x} \psi(A)$ (part (c)) is not equal to $\gamma_{x}\left(\operatorname{id} \bigvee \gamma_{x} \psi\right)(A)$ (part (d)).

Property 1. Extensive and anti-extensive mappings are adjacency stable.

Property 2. The class of adjacency stable connected operators is closed under the sup, the inf and the sequential composition operations.

If $\psi$ is an adjacency stable connected operator, then, for all $A \in \mathcal{P}(E)$ : (a) $x \notin A, x \in \psi(A) \Rightarrow \mathrm{D}_{x}(A) \leq \psi(A)$; (b) $x \in A, x \notin \psi(A) \Rightarrow \mathrm{D}_{x}(A) \leq$ $\complement \psi(A)$.

\section{Levelings and set levelings}

A leveling [20-23] is defined next.

Definition 3. An image $g$ is a leveling of an input image $f$ if and only if:

$$
\forall(p, q) \text { neighboring pixels : } g_{p}>g_{q} \Rightarrow f_{p} \geq g_{p} \text { and } g_{q} \geq f_{q} \text {. }
$$

The previous definition of leveling is that in [20, Definition 4 (p. 193)] [23, Definition 2.2 (p. 4)]. A more general and complex definition is introduced in [22, Definition 10 (p. 62)], but a leveling as established by Expression 3 is also a leveling as defined in [22, Definition 10 (p. 62)]. Moreover, we focus on set operators.

Set levelings are those defined in the set or binary framework. Expression 3 will be particularized for the set or binary case in Section 5.2. 
Proceedings of the $8^{\text {th }}$ International Symposium on Mathematical Morphology,

Rio de Janeiro, Brazil, Oct. 10-13, 2007, MCT/INPE, v. 1, p. 215-226.

http://urlib.net/dpi.inpe.br/ismm@80/2007/03.01.11.36

\section{Relationships between adjacency stable connected operators and set levelings}

Both adjacency stable connected operators and levelings impose certain constraints on the input and output variations, which will analyzed next. Then, the relationships between these types of operators will be discussed.

\subsection{Adjacency stable connected operators: input/out- put variations restriction}

First, we will formalize the neighborhood relationship between two pixels using $\gamma_{x}$. As in Section 4 (Definition 3), we will use the symbols $p$ and $q$ to refer to a pair of pixels that are neighbors. Two pixels $p$ and $q$ are neighbors to each other if and only if they satisfy

$$
\gamma_{p}(\{p, q\})=\gamma_{q}(\{p, q\})=\{p, q\} .
$$

The next proposition, which states the restrictions imposed on the values of neighboring pixels between an input $I$ and the output $I^{\prime}$ of an adjacency stable connected operator, follows directly from Definition 2.

Property 3. Let $A$ be an input set. Let $\psi: \mathcal{P}(E) \Rightarrow \mathcal{P}(E)$ be an adjacency stable connected operator. If $p$ and $q$ are neighbors to each other (i.e., $p$ and $q$ satisfy equation 4), or if they belong to adjacent flat zones, the adjacency stability of $\psi$ implies the following restrictions between the input $A$ and output $\psi(A)$ sets:

$$
\begin{aligned}
& \text { (a) } \gamma_{p}(A)=\gamma_{q}(A) \Rightarrow \gamma_{p} \psi(A)=\gamma_{q} \psi(A), \\
& \text { (b) } p \in A, q \notin A \Rightarrow\left\{\begin{array}{c}
p \in \psi(A), q \notin \psi(A) \\
\text { or } \\
\gamma_{p} \psi(A)=\gamma_{q} \psi(A) .
\end{array}\right.
\end{aligned}
$$

There is a case symmetric to (b) above (interchanging $p$ and $q$ in (b)) not shown.

The cases not covered in Expression 5 are: (i) $p \in A, q \notin A$ and $p \notin$ $\psi(A), q \in \psi(A)$ ); and (ii) $p \notin A, q \in A$ and $p \in \psi(A), q \notin \psi(A)$. (Cases (i) and (ii) are symmetric to each other, interchanging $p$ and $q$.) Clearly, these cases do not satisfy Equation 2 of Definition 2. Let us prove that for one case. We can prove that the case $p \notin A, q \in A$ and $p \in \psi(A), q \notin \psi(A))$ does not satisfy Equation 2 . We have that:

- $\gamma_{p}(\mathrm{id} \bigvee \psi)(A)=\gamma_{p}(A \bigvee \psi(A))=\gamma_{p} \psi(A) \bigvee \gamma_{q}(A)$,

- $\gamma_{p}(A) \bigvee \gamma_{p} \psi(A)=\gamma_{p} \psi(A)$,

and $\gamma_{p} \psi(A) \bigvee \gamma_{q}(A)>\gamma_{p} \psi(A)$, since $q \in A, \gamma_{q}(A) \neq \emptyset$, and $\gamma_{q}(A) \not{Z}$ $\gamma_{p} \psi(A)$.

We can straightforwardly extend Property 3 to binary functions (which can be considered as equivalent to sets). Let us assume that the binary 
Proceedings of the $8^{\text {th }}$ International Symposium on Mathematical Morphology,

Rio de Janeiro, Brazil, Oct. 10-13, 2007, MCT/INPE, v. 1, p. 215-226.

http://urlib.net/dpi.inpe.br/ismm@80/2007/03.01.11.36

values are 0 and 1 . Let $I$ and $I^{\prime}$ be, respectively, an input image and the output of an adjacency stable connected operator $\psi$ (i.e., $I^{\prime}=\psi(I)$ ).

Binary functions case: If $p$ and $q$ are neighbors to each other (or if they belong to adjacent flat zones), then:

$$
\begin{aligned}
& \text { (a) } I_{p}=I_{q} \Rightarrow I_{p}^{\prime}=I_{q}^{\prime}, \\
& \text { (b) } I_{p}=1, I_{q}=0 \Rightarrow\left\{\begin{array}{c}
I_{p}^{\prime}=1, I_{q}^{\prime}=0 \\
\text { or } \\
I_{p}^{\prime}=I_{q}^{\prime} .
\end{array}\right.
\end{aligned}
$$

Note: the case symmetric to (b) (interchanging $p$ and $q$ in (b)) is not shown.

The cases ruled out are: (i) $I_{p}=1, I_{q}=0$, and $I_{p}^{\prime}=0, I_{q}^{\prime}=1$; and (ii) $I_{p}=0, I_{q}=1$, and $I_{p}^{\prime}=1, I_{q}^{\prime}=0$. (Cases (i) and (ii) are symmetric to each other, interchanging $p$ and $q$.)

\subsection{Set levelings: input/output variation restriction}

Definition 3 can of course be applied to binary images (or, equivalently, sets). If we particularize for binary images then we have the following, concerning Expression 3. Similarly as in Section 5.1, $I$ and $I^{\prime}$ denote the input and output images (i.e., $I$ and $I^{\prime}$ have been substituted for $f$ and $g$, respectively, in Expression 3). An inequality such as $I_{p}^{\prime}>I_{q}^{\prime}$ can only occur when there is a discontinuity where $I_{p}^{\prime}$ and $I_{q}^{\prime}$ are 1 and 0 , respectively. Then, Expression 3 can only be:

$$
1>0 \Rightarrow 1 \geq 1 \text { and } 0 \geq 0,
$$

i.e., $I_{p}$ has to be 1 , and $I_{q}$ must be 0 .

Therefore, if $I_{p}^{\prime}=1$ and $I_{q}^{\prime}=0$, the case where $I_{p}=0$ and $I_{q}=1$ is excluded.

\subsection{Discussion}

We can now precisely state the relationships between stable connected operators and set levelings: (a) Both adjacent stable connected operators and set levelings impose restrictions on the input/output variations. (b) The imposed restrictions are identical in both cases: if $I_{p}^{\prime}=1$ and $I_{q}^{\prime}=0$, then $I_{p}$ and $I_{q}$ must be 1 and 0 , respectively.

Thus, the set leveling concept and the adjacency stable connected operator concept are equivalent. The adjacency stable connected operator notion $[6,10,11]$ is prior in time to levelings $[20,21,23]$ [22].

A final question can arise: can the adjacency stable connected operator concept be applied to a gray-level framework? The answer is affirmative, 
Proceedings of the $8^{\text {th }}$ International Symposium on Mathematical Morphology,

Rio de Janeiro, Brazil, Oct. 10-13, 2007, MCT/INPE, v. 1, p. 215-226.

http://urlib.net/dpi.inpe.br/ismm@80/2007/03.01.11.36

we can directly extend such input and output variation restrictions to flat gray-level connected operators that commute with thresholding, where the operator is level-by-level connected and where Equation 2 must hold for all sections of the input and output gray-level functions. Let $I$ and $I^{\prime}$ be, respectively, an input image and the output of a flat gray-level adjacency stable connected operator $\psi$ (i.e., $I^{\prime}=\psi(I)$ ).

Gray-level (non-binary) functions case: If $p$ and $q$ are neighbors to

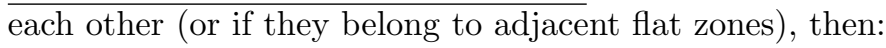

$$
\begin{aligned}
& \text { (a) } I_{p}=I_{q} \Rightarrow I_{p}^{\prime}=I_{q}^{\prime}, \\
& \text { (b) } I_{p}>I_{q} \Rightarrow\left\{\begin{array}{c}
I_{p}^{\prime}>I_{q}^{\prime} \\
\text { or } \\
I_{p}^{\prime}=I_{q}^{\prime} .
\end{array}\right.
\end{aligned}
$$

Note: the case symmetric to (b) is not shown.

The case ruled out is: $I_{p}<I_{q}$, and $I_{p}^{\prime}>I_{q}^{\prime}$ (as well as the symmetric one: $I_{p}>I_{q}$, and $I_{p}^{\prime}<I_{q}^{\prime}$ ). This case is also excluded by Expression 3 of levelings. Moreover, disregarding trivial cases (such as those where, processing level by level, $\psi(\emptyset)=E$ or $\psi(E)=\emptyset$ ), in flat morphological (increasing) connected operators the variation range of the output is equal or smaller than that of the input. Otherwise, the flat zone inclusion relationship of connected operators would not necessarily hold between sections at same level of the input and output images. For example, in the case where $I_{p}>I_{q}$ and $I_{p}^{\prime}>I_{q}^{\prime}$, the next gradation would exist : $I_{p} \geq I_{p}^{\prime}>I_{q}^{\prime} \geq I_{q}$.

Now that the equivalence of adjacency stable connected operators and set levelings has been established, researchers know that the adjacency stable connected operator properties also hold for (flat) levelings. Let us point out that the properties included in Section 3 (Lemma 1 and Properties 1 and 2), altogether with the adjacency stability equation 2, are useful for manipulating expressions composed of connected operators, especially when they are also connected-component local (i.e., they treat grains or pores independently from the rest) $[6,10,11]$. In fact, they will be employed in a proof in the following section.

\section{On commutative properties of open-close and close-open filters for connected operators}

In this section we will first comment on an interesting commutative property that has been presented elsewhere, and on some of its implications and limits. Afterwards, we will also present another commutative property that is satisfied by certain alternated connected filters, particularly by attribute alternated filters. 
Proceedings of the $8^{\text {th }}$ International Symposium on Mathematical Morphology,

Rio de Janeiro, Brazil, Oct. 10-13, 2007, MCT/INPE, v. 1, p. 215-226.

http://urlib.net/dpi.inpe.br/ismm@80/2007/03.01.11.36

\subsection{Marker-based operators}

Let us define the "||" relationship presented in [33, Definition 7.3]:

Definition 4. Let $A$ and $B$ be, respectively, a grain (a connected set) and a set. We say that $A \| B$ if $A$ and $B$ have a non-empty intersection or are adjacent.

Now, we are going to define two operators, $\bar{\gamma}$ and $\varphi$, based on makers that are presented in [33, p. 176]. Besides one normal input set, those operators use a second one, which is a marker set.

Definition 5. Let $A$ and $M$ be two sets. The connected operator $\bar{\gamma}$ of a set $A$ based on marker $M$, symbolized by $\bar{\gamma}(A, M)$, is defined as:

$$
\bar{\gamma}(A, M)=\bigcup\left\{\gamma_{x}(A): \gamma_{x}(A) \| M\right\} .
$$

Note that Definition 5 does not define $\bar{\gamma}(A)$ but $\bar{\gamma}(A, M)$.

Definition 6. Let $A$ and $M$ be two sets. The connected operator $\underline{\varphi}$ of a set $A$ based on marker $N$, symbolized by $\underline{\varphi}(A, N)$, is defined as:

$$
\complement[\underline{\varphi}(A, \complement N)]=\bigcup\left\{\gamma_{x}(\complement A), x \in E: \gamma_{x}(\complement A) \| N .\right\}
$$

In [33], it is established that, under some connectivity considerations, there exists a commutative property for $\bar{\gamma}$ and $\varphi$ [33, Theorem 7.3]:

$$
\bar{\gamma}(\underline{\varphi}(A, \complement N), M)=\underline{\varphi}\left(\bar{\gamma}(A, \bar{M}), \complement_{N}\right) .
$$

It is indicated in [33] that $\bar{\gamma}(\varphi(A, \complement N), M)$ (or $\varphi(\bar{\gamma}(A, M), \complement N))$ is a leveling and that is a strong filter. Moreover, in [22,23], it is mentioned that levelings are strong filters (see [23, Section 3.4 (p. 9)] and [22, Section 4.4.1 (p. 67)]), and the discussion refers to a commutative expression similar to the aforementioned one. This should be clarified, because it seems there could be some confusion about levelings (Definition 3), particularly about whether all levelings can be formulated as sequential compositions of an opening and a closing, and vice-versa.

There is an important remark, which concerns the main cause of possible misconceptions, to be made about Equation 11: the computation of the markers is not considered. Thus, saying that an operation can be expressed as in Equation 11 does not necessarily imply that such an operation can be expressed as a sequential composition of an opening and a closing, and vice-versa, when the marker computation is considered. For example, in $\bar{\gamma}(\varphi(A, \complement N), M)$, the marker $M$ of $\bar{\gamma}$ is not affected by the result of the previously applied $\underline{\varphi}$; that is, the $M$ marker can be considered as an input. This is not how we usually think about openings and closings. In fact, when the marker computation is adequately considered, not all levelings (Definition 3) can be expressed as a sequential composition of an opening and a closing, and vice-versa; and it would not be 
the case that all levelings are strong filters. (See [9] [8] for further discussion about the strong property of connected alternated filters.)

Next section will present a commutative property for certain connected filters that does not raise that objection but where the underlying marker is, as usual, not considered fixed.

\subsection{A commutative property}

We present a commutative property for a certain type of connected alternated filters, particularly for alternated attribute filters, which are strong filters composed of an attribute opening [31,34] [38] [35] and an attribute closing. Area openings and closings are examples of attribute openings and closings, respectively.

Let $\tilde{\gamma}$ and $\tilde{\varphi}$ denote, respectively an attribute opening and closing. In the following, unlike in Equation 11, when we write $\tilde{\varphi} \tilde{\gamma}$ it is clear that the criterion (and associated marker) of $\tilde{\varphi}$ is applied to the output computed by the previous $\tilde{\gamma}$. We have the following property:

Property 4. An attribute alternated filter $\tilde{\varphi} \tilde{\gamma}$ can be expressed as a commutative sequential composition of an opening and a closing as follows:

$$
\tilde{\varphi} \tilde{\gamma}=\tilde{\gamma}(\operatorname{id} \bigvee \tilde{\varphi} \tilde{\gamma})=(\operatorname{id} \bigvee \tilde{\varphi} \tilde{\gamma}) \tilde{\gamma}
$$

Proof. There are two equalities to consider.

(a) Let $A$ be a set. Since $\tilde{\gamma}$ is connected-component local we have $\tilde{\gamma}=$ $\bigvee_{x} \gamma_{x} \tilde{\gamma}=\bigvee_{x} \tilde{\gamma} \gamma_{x}$. Thus, $\tilde{\gamma}(\mathrm{id} \bigvee \tilde{\varphi} \tilde{\gamma})(A)=\bigvee_{x} \gamma_{x} \tilde{\gamma}(\mathrm{id} \bigvee \tilde{\varphi} \tilde{\gamma})(A)=$ $\bigvee_{x} \tilde{\gamma} \gamma_{x}(\mathrm{id} \bigvee \tilde{\varphi} \tilde{\gamma})(A)$. From Property 1 and Property $2, \tilde{\varphi} \tilde{\gamma}$ is adjacency stable, and, from Lemma 1, $\tilde{\varphi} \tilde{\gamma}(A)$ and $A \backslash \tilde{\varphi} \tilde{\gamma}(A)$ are not adjacent $[6,10]$. Then,

$$
\tilde{\gamma} \gamma_{x}(\operatorname{id} \bigvee \tilde{\varphi} \tilde{\gamma})(A)= \begin{cases}\tilde{\gamma} \gamma_{x}(A)=\emptyset, & x \in A \backslash \tilde{\varphi} \tilde{\gamma}(A) \\ \tilde{\gamma} \gamma_{x} \tilde{\varphi} \tilde{\gamma}(A), & x \in \tilde{\varphi} \tilde{\gamma}(A)\end{cases}
$$

Thus, $\bigvee_{x} \tilde{\gamma} \gamma_{x} \tilde{\varphi} \tilde{\gamma}=\bigvee_{x} \gamma_{x} \tilde{\gamma} \tilde{\varphi} \tilde{\gamma}=\tilde{\gamma} \tilde{\varphi} \tilde{\gamma}$. Finally, $\tilde{\gamma} \tilde{\varphi} \tilde{\gamma}=\tilde{\varphi} \tilde{\gamma}$ (since $\tilde{\varphi} \tilde{\gamma} \leq$ $\tilde{\gamma} \tilde{\varphi}$ and $\tilde{\gamma} \tilde{\varphi} \tilde{\gamma}=\tilde{\varphi} \tilde{\gamma}[28,34])$.

(b) $(\mathrm{id} \bigvee \tilde{\varphi} \tilde{\gamma}) \tilde{\gamma}=\tilde{\gamma} \bigvee \tilde{\varphi} \tilde{\gamma} \tilde{\gamma}=\tilde{\gamma} \bigvee \tilde{\varphi} \tilde{\gamma}=\tilde{\varphi} \tilde{\gamma}$.

Notes: (a) (id $\bigvee \tilde{\varphi} \tilde{\gamma}$ ) is a closing (and different from $\tilde{\varphi}$ ). (b) Property 4 is different from [17, Proposition 10.2]. (c) This proof also provides an example of using adjacent stable connected operators properties to manipulate expressions. (d) Concerning filter expressions and decompositions, see also [7].

\section{Conclusion}

This paper has focused on connected morphological operators. First, the relationship between adjacency stable connected operators and set levelings has been investigated, and a close relationship, an equivalence, has been 
identified. This is important to establish how and when this concept has been introduced, and to clarify its origin. In addition, properties satisfied by one class of operators can be applied to the other equivalent one.

Second, this work has analyzed a commutative property previously presented, as well as some of its implications and limits. The paper has presented also a commutative property for attribute alternated filters, in which, as is usually the case and unlike in the other commutative property analyzed, the underlying marker computation is taken into account.

\section{Acknowledgements}

The author wants to thank the comments of Dr. Ronse about strong connectivities, as well as the remarks of an anonymous reviewer.

\section{References}

[1] G. J. F. Banon, Formal introduction to digital image processing, INPE, São José dos Campos, 2000. Available from: <http://urlib.net/dpi.inpe.br/banon/1999/ 06.21.09.31>. Access in: 2007-02-17.

[2] U. Braga-Neto, Multiscale connected operators, Journal of Mathematical Imaging and Vision 22 (2005), no. 2 - 3, 199-216.

[3] U. Braga-Neto and J. Goutsias, A multiscale approach to connectivity, Computer Vision and Image Understanding 89 (2003), no. 1, 70-107.

[4] - A theoretical tour of connectivity in image processing and analysis, Journal of Mathematical Imaging and Vision 19 (2003), no. 1, 5-31.

[5] E. J. Breen and R. Jones, Attribute openings, thinnings, and granulometries, Computer Vision and Image Understanding 64 (1996), no. 3, 377-389.

[6] J. Crespo, Morphological connected filters and intra-region smoothing for image segmentation, Ph.D. Thesis, 1993.

[7] J. Crespo and V. Maojo, New results on the theory of morphological filters by reconstruction, Pattern Recognition 31 (1998), no. 4, 419-429.

[8] , The strong property of morphological connected alternated filters, submitted for publication (2007).

[9] J. Crespo, V. Maojo, J. A. Sanandrés, H. Billhardt, and A. Muñoz, On the strong property of connected open-close and close-open filters, Discrete geometry for computer imagery, 2002, pp. 165-174.

[10] J. Crespo and R. W. Schafer, Locality and adjacency stability constraints for morphological connected operators, Journal of Mathematical Imaging and Vision 7 (1997), no. 1, 85-102.

[11] J. Crespo, J. Serra, and R. W. Schafer, Image segmentation using connected filters, Workshop on mathematical morphology, Barcelona, 1993, pp. 52-57.

[12] _ Theoretical aspects of morphological filters by reconstruction, Signal Processing 47 (1995), no. 2, 201-225.

[13] E. R. Dougherty and R. A. Lotufo, Hands-on morphological image processing, SPIE Press, Bellingham, 2003.

[14] L. Garrido, P. Salembier, and D. Garcia, Extensive operators in partition analysis for image sequence analysis, Signal Processing 66 (1998), no. 2, 157-180. 
Proceedings of the $8^{\text {th }}$ International Symposium on Mathematical Morphology,

Rio de Janeiro, Brazil, Oct. 10-13, 2007, MCT/INPE, v. 1, p. 215-226.

http://urlib.net/dpi.inpe.br/ismm@80/2007/03.01.11.36

[15] C. Giardina and E. Dougherty, Morphological methods in image and signal processing, Prentice-Hall, Englewood Clliffs, 1988.

[16] H. Heijmans, Morphological image operators (advances in electronics and electron physics; Series Editor: P. Hawkes), Academic Press, Boston, 1994.

[17] _ Connected morphological operators for binary images, Computer Vision and Image Understanding 73 (1999), 99-120.

[18] R. Jones, Connected filtering and segmentation using component trees, Computer Vision and Image Understanding 75 (1999), no. 3, 215-228.

[19] G. Matheron, Random sets and integral geometry, Wiley, New York, 1975.

[20] F. Meyer, From connected operators to levelings, Mathematical morphology and its applications to image and signal processing, 1998, pp. 191-198.

[21] _ The levelings, Mathematical morphology and its applications to image and signal processing, 1998, pp. 199-206.

[22] _ Levelings, image simplification filters for segmentation, Journal of Mathematical Imaging and Vision 20 (2004), no. 1-2, 59-72.

[23] F. Meyer and P. Maragos, Nonlinear scale-space representation with morphological levelings, Journal of Visual Communication and Image Representation 11 (2000), no. 3, 245-265.

[24] C. Ronse, Set-theoretical algebraic approaches to connectivity in continuous or digital spaces, Journal of Mathematical Imaging and Vision 8 (1998), no. 1, 41-58.

[25] Guest editorial, Journal of Mathematical Imaging and Vision 22 (2005), no. 2 - 3, 103-105.

[26] C. Ronse and J. Serra, Geodesy and connectivity in lattices, Fundamenta Informaticae 46 (2001), no. 4, 349-395.

[27] P. Salembier and L. Garrido, Binary partition tree as an efficient representation for image processing, segmentation, and information retrieval, IEEE Transactions on Image Processing 9 (2000), no. 4, 561-575.

[28] P. Salembier and J. Serra, Flat zones filtering, connected operators, and filters by reconstruction, IEEE Transactions on Image Processing 4 (1995), no. 8, 1153-1160.

[29] M. Schmitt and J. Mattioli, Morphologie mathématique, Masson, Paris, 1993.

[30] J. Serra, Mathematical morphology. Volume I, Academic Press, London, 1982.

[31] (ed.), Mathematical morphology. Volume II: Theoretical Advances, Academic Press, London, 1988.

[32] Connectivity in complete lattices, Journal of Mathematical Imaging and Vision 9 (1998), no. 3, 231-251.

[33] Connections for sets and functions, Fundamenta Informaticae 41 (2000), no. 1-2, 147-186.

[34] J. Serra and P. Salembier, Connected operators and pyramids, Proceedings of SPIE, non-linear algebra and morphological image processing, san diego, 1993, pp. 65-76.

[35] P. Soille, Morphological image analysis: Principles and applications, 2nd., SpringerVerlag, Berlin-Heidelberg-New York, 2003.

[36] I. R. Terol and D. Vargas, A study of openings and closings with reconstruction criteria, Proc. of international symposium on mathematical morphology (ISMM) 2002, 2002.

[37] D. Vargas-Vázquez, J. Crespo, and V. Maojo, Morphological image reconstruction with criterion from labelled markers, Discrete geometry for computer imagery, 2003, pp. $475-484$.

[38] L. Vincent, Morphological grayscale reconstruction in image analysis: Applications and efficient algorithms, IEEE Transactions on Image Processing 2 (1993), 176-201. 